\title{
Wheal Longest Diameter
}

National Cancer Institute

\section{Source}

National Cancer Institute. Wheal Longest Diameter. NCI Thesaurus. Code C112427.

The longest diameter of the flat, circular, slightly raised area that forms around the site of an antigenic challenge to the skin. 